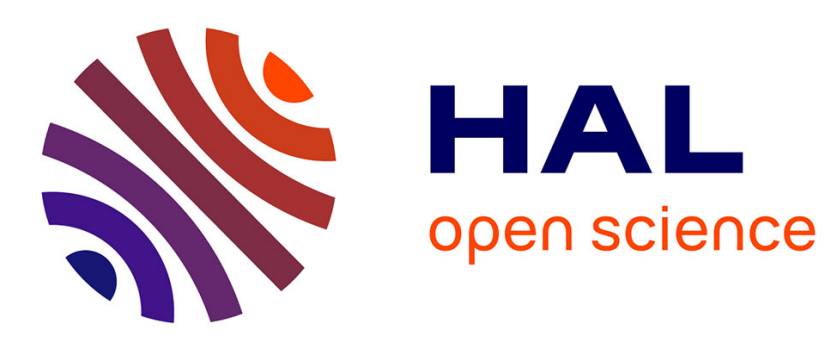

\title{
Expander graphs and sieving in combinatorial structures
}

Florent Jouve, Jean-Sébastien Sereni

\section{To cite this version:}

Florent Jouve, Jean-Sébastien Sereni. Expander graphs and sieving in combinatorial structures. Journal of the Australian Mathematical Society, 2018, 105 (1), pp.79-102. 10.1017/S1446788717000234 . hal-00693334v3

\section{HAL Id: hal-00693334 \\ https://hal.science/hal-00693334v3}

Submitted on 6 Jan 2017

HAL is a multi-disciplinary open access archive for the deposit and dissemination of scientific research documents, whether they are published or not. The documents may come from teaching and research institutions in France or abroad, or from public or private research centers.
L'archive ouverte pluridisciplinaire HAL, est destinée au dépôt et à la diffusion de documents scientifiques de niveau recherche, publiés ou non, émanant des établissements d'enseignement et de recherche français ou étrangers, des laboratoires publics ou privés. 


\title{
EXPANDERS GRAPHS AND SIEVING IN COMBINATORIAL STRUCTURES
}

\author{
FLORENT JOUVE AND JEAN-SÉBASTIEN SERENI
}

\begin{abstract}
We prove a general large sieve statement in the context of random walks on subgraphs of a given graph. This can be seen as a generalization of previously known results where one performs a random walk on a group enjoying a strong spectral gap property. In such a context the point is to exhibit a strong uniform expansion property for a suitable family of Cayley graphs on quotients. In our combinatorial approach, this is replaced by a result of Alon-Roichman about expanding properties of random Cayley graphs. Applying the general setting we show e.g., that with high probability (in a strong explicit sense) random coloured subsets of integers contain monochromatic (non-empty) subsets summing to 0 , or that a random coloring of the edges of a complete graph contains a monochromatic triangle.
\end{abstract}

\section{INTRODUCTION}

The relevance of using families of expander graphs for studying objects or solving problems coming from a broad variety of mathematical areas has been emphasized in numerous ways in the recent years. Notably the combination of sieving arguments together with expansion properties has proved particularly efficient. Let us mention the groundbreaking work [4] where the mix of such techniques enabled the authors to detect almost primes in a variety of non-Abelian situations. A different kind of sieve together with the same expansion properties have also been exploited in the context of group theory [11], or to obtain quantitative results in the probabilistic Galois theory of arithmetic groups [7]. In the sieving processes used in the aforementioned works, one is naturally led to a crucial step where some spectral gap property is needed. Deep results about the groups involved then come into play. Typically the required properties are provided by recent breakthroughs in algebraic combinatorics that have led to strong forms of Lubotzky's Property $(\tau)$ (so-called superstrong approximation, a culminating point of the study of which is the work by Golsefidy and Varjú [15]). Indeed, the spectral gap result needed can be tautolgically interpreted as a property of expansion of a certain family of graphs.

The goal of the present paper is to establish a general large sieve inequality in a purely combinatorial setting. More precisely, we develop an axiomatic version of sieve in the context of countable families of Cayley graphs, which are randomly generated via a random walk. This can be viewed as a generalisation of the framework used e.g. in [8, Chap. 7] or [7] to a situation where spectral gap properties on groups are no longer available. To circumvent the lack of algebraic structure we will exploit the fact that random graphs are "good expanders". Precisely we will use a result of Alon-Roichman [3] according to which a family of random Cayley graphs is very likely to form a good family of expanders. However, further difficulties arise: contrary to the usual situation, expansion properties are not sufficient to ensure good enough cancellation in the correlation sums appearing. To obtain the required cancellation we introduce structural properties and use concentration arguments. To the best of our knowledge, this point of view is new within sieving contexts and it seems to us that it might lead to new uses and theoretical study of sieves in a combinatorial setting. 
After describing our general setting and proving our main result (Theorem 1.1), we briefly present some concrete uses of our result to specific questions.

We describe several applications of our method to the study of typical properties of subgraphs of a given graph. To produce random elements for which we want to test if some given property holds, we perform a random walk on the family of graphs studied, (cf. also [7]). Another approach could consist in quantifying the proportion of elements that satisfy an expected property among a finite subset of the family of graphs considered. For the applications we have in mind this question would in fact be much easier. As a matter of fact we do need to quantify proportions of "good" elements as part of our sieving process.

The paper is organized as follows. In Section 1 we state and prove the main result and we emphasize the way in which Alon-Roichman's theorem enables us to work in a setting which is combinatorial in nature (whereas earlier works such as [8, Chap. 7] required a more algebraic framework). The rest of the paper is devoted to applications of the main result. Let us end the introduction by giving (rough) statements for some of the concrete consequences of our main theoretical result (Theorem 1.1). We conclude the paper with remarks on further questions that may be of interest and that can be successfully investigated via our method. We notably state a Ramsey type result (together with a sketch of proof) obtained by suitably adapting the arguments used in the second application.

Notation. If $X$ is a finite set, then $\# X$ and $|X|$ synonymously denote the cardinality of $X$.

If $X$ is a finite graph, then $\operatorname{Adj}(X)$ is the adjacency operator sending a $\mathbf{C}$-valued function on the vertices of $X$ to the function $\left(x \mapsto \sum_{y} f(y)\right)$, where the sum is over the neighbors $y$ of the vertex $x$. If $X$ is moreover $d$-regular (that is, every vertex of $X$ has degree $d$ ), then the normalized adjacency operator is $\frac{1}{d} \cdot \operatorname{Adj}(X)$.

If $G$ is a group and $S \subset G$, then $X(G, S)$ is the Cayley graph on $G$ with edge set $S \cup S^{-1}:=$ $\left\{s \in G: s \in S\right.$ or $\left.s^{-1} \in S\right\}$. If $G$ is finite and Abelian, then $\hat{G}$ is the character group of $G$. If $x$ is a non-negative real number, then $\lceil x\rceil$ and $\lfloor x\rfloor$ are the least integer greater than or equal to $x$ and the greatest integer smaller than or equal to $x$, respectively. If $R$ is a positive integer, then $[R]$ is the set $\{1, \ldots, R\}$. Given a probability space $(\Omega, \Sigma, \boldsymbol{P})$ and two events $A$ and $B$ such that $\boldsymbol{P}(B) \neq 0$, we let $\boldsymbol{P}(A \mid B)$ be the conditional probability $\boldsymbol{P}(A \cap B) / \boldsymbol{P}(B)$. If $f$ and $g$ are two real-valued functions defined on a set $\mathcal{D}$ and depending on a set $\mathcal{P}$ of parameters, then $f(x) \ll \mathcal{P}_{0} g(x)$ means that there exists a positive constant $C$ depending only on the subset $\mathcal{P}_{0} \subseteq \mathcal{P}$ such that $|f(x)| \leqslant C|g(x)|$ whenever $x \in \mathcal{D}$.

\section{The GEneral SETting AND A LARGE SIEVE FOR GRAPHS}

1.1. Random walk large sieve: statement of the main result. Stating our main result requires some definitions and a precise description of the general setting. Let $G$ be an Abelian group (in this section, the group law is noted multiplicatively) and $\Lambda \subset \mathbf{N}$ be a (non necessarily finite) set of indices. We suppose we are given a family $\left(H_{\ell}\right)_{\ell \in \Lambda}$ of subgroups of $G$ such that for each $\ell$ the index $n_{\ell}:=\left[G: H_{\ell}\right]$ is finite. We let $\rho_{\ell}: G \rightarrow G / H_{\ell}$ be the canonical projection. If $\ell$ and $\ell^{\prime}$ are two distinct elements of $\Lambda$, we define $\rho_{\ell, \ell^{\prime}}: G \rightarrow G / H_{\ell} \times G / H_{\ell^{\prime}}$ by $g \mapsto\left(\rho_{\ell}(g), \rho_{\ell^{\prime}}(g)\right)$.

We fix once and for all a probability space $(\Omega, \Sigma, \boldsymbol{P})$ and an arbitrarily small real $\delta \in(0,1)$. We further set

$$
\psi(\delta):=2((2-\delta) \log (2-\delta)+\delta \log \delta)^{-1} .
$$

For each $\ell \in \Lambda$, we define the quantity

$$
\kappa\left(b_{\ell}, \ell ; \delta\right):=\left\lceil\psi(\delta)\left(\log n_{\ell}+b_{\ell}+\log 2\right)\right\rceil,
$$

where $b:=\left(b_{\ell}\right)$ is a parameter (a sequence of positive real numbers). 
Now let $s_{1}^{(\ell)}, \ldots, s_{\kappa\left(b_{\ell}, \ell ; \delta\right)}^{(\ell)}$ be independent identically distributed random variables taking values in $G / H_{\ell}$. The random walk on $G$ we want to consider is obtained by lifting the sets $\left\{s_{1}^{(\ell)}, \ldots, s_{\kappa\left(b_{\ell}, \ell ; \delta\right)}^{(\ell)}\right\}$ (and their "inverses" so that all the graphs considered are then undirected) to $G$. To that purpose we define the random variable

$$
S_{\ell}\left(b_{\ell}, \delta\right):=\left\{s_{1}^{(\ell)}, \ldots, s_{\kappa\left(b_{\ell}, \ell ; \delta\right)}^{(\ell)}\right\} \cup\left\{\left(s_{1}^{(\ell)}\right)^{-1}, \ldots,\left(s_{\kappa\left(b_{\ell}, \ell ; \delta\right)}^{(\ell)}\right)^{-1}\right\},
$$

which takes values in the set of subsets of $G / H_{\ell}$. For every index $\ell \in \Lambda$ and every integer $m \in\left\{1, \ldots, \kappa\left(b_{\ell}, \ell ; \delta\right)\right\}$, we need to choose a representative $\tilde{s}_{m}^{(\ell)} \in G$ of $s_{m}^{(\ell)}$. The particular representative we choose is imposed by the following condition of admissibility. (As we shall establish later on, for each family of subgroups there exists at most one admissible local sequence in the sense of Definition 1.)

Definition 1. Let $\left(H_{\ell}\right)_{\ell \in \Lambda}$ be a fixed family of subgroups of finite index of $G$ and, for each $\ell \in \Lambda$, let $R_{\ell}$ be a set of representatives of $G / H_{\ell}$. The sequence $\left(H_{\ell}, R_{\ell}\right)_{\ell \in \Lambda}$ is called an admissible local sequence for $G$ if

(i) $\bigcap_{\ell \in \Lambda} H_{\ell}=\{1\}$; and

(ii) $\forall \ell, \ell^{\prime} \in \Lambda, \quad \ell \neq \ell^{\prime} \Rightarrow R_{\ell} \subseteq H_{\ell^{\prime}}$.

Let us assume that the sequence $\left(H_{\ell}\right)$ of subgroups of $G$ is such that there is an admissible local sequence $\left(H_{\ell}, R_{\ell}\right)$ for $G$. For each $\ell$ and each $m \in\left\{1, \ldots, \kappa\left(b_{\ell}, \ell ; \delta\right)\right\}$, we choose the unique representative $\tilde{s}_{m}^{(\ell)}$ of $s_{m}^{(\ell)}$ in $R_{\ell}$. The aforementioned uniqueness of an admissible local sequence ensures (see Lemma 1.2) that this defines in a unique way elements $\tilde{s}_{m}^{(\ell)}$ in $G$. Next we set

$$
\tilde{S}_{\ell}(b, \delta):=\left\{\tilde{s}_{1}^{(\ell)}, \ldots, \tilde{s}_{\kappa\left(b_{\ell}, \ell ; \delta\right)}^{(\ell)}\right\} \cup\left\{\left(\tilde{s}_{1}^{(\ell)}\right)^{-1}, \ldots,\left(\tilde{s}_{\kappa\left(b_{\ell}, \ell ; \delta\right)}^{(\ell)}\right)^{-1}\right\} .
$$

The subset of $G$ we use to perform a random walk on $G$ is

$$
S(b, \delta):=\prod_{\ell \in \Lambda}^{*}\left(\{1\} \cup \tilde{S}_{\ell}\left(b_{\ell}, \delta\right)\right) .
$$

Let us explain precisely what the notation means. If $A_{1}, \ldots, A_{k}$ are $k$ subsets of $G$, the product $\prod_{i=1}^{k} A_{i}$ is the subset $\left\{a_{1} \ldots a_{k}: a_{i} \in A_{i}\right\}$ of $G$. Here the symbol $\prod^{*}$ means that for all $\ell$ but finitely many of them the $\ell$-th factor picked equals 1 . Finally $S(b, \delta)$ is not seen as a random variable but as the product over $\ell$ of elements either equal to 1 or picked in $\tilde{S}_{\ell}\left(b_{\ell}, \delta\right)$ evaluated at a common $\omega \in \Omega$. In other words we fix once and for all an element $\omega$ of $\Omega$; picking an element of $S(b, \delta)$ amounts to picking 1 or an element of some $\tilde{S}_{\ell}\left(b_{\ell}, \delta\right)(\omega)$, and then computing the product of these elements.

With notation as above, we perform the following random walk on $G$. It is defined the same way as in $[8$, Chap. 7$]$.

$$
\left\{\begin{array}{l}
X_{0}=g_{0} \\
X_{k+1}=X_{k} \xi_{k+1} \quad \text { for } k \geqslant 0,
\end{array}\right.
$$

where $g_{0}$ is a fixed element in $G$ and the steps $\xi_{k}$ are independent, identically distributed random variables with distribution

$$
\boldsymbol{P}\left(\xi_{k}=s\right)=\boldsymbol{P}\left(\xi_{k}=s^{-1}\right)=p_{s}=p_{s^{-1}}
$$

for every $k$ and every $s \in S(b, \delta)$, and where $\left(p_{s}\right)_{s}$ is a sequence of positive real numbers indexed by $S(b, \delta)$ such that

$$
\sum_{s \in S(b, \delta)} p_{s}=1
$$


Of course the random walk depends on the parameters $b=\left(b_{\ell}\right)_{\ell}$ and $\delta$. If $\Lambda$ is finite, the most natural such random walk is certainly the one defined by uniformly distributing the steps, that is, $p_{s}:=\# S(b, \delta)^{-1}$ for every $s \in S(b, \delta)$. In general, we require that the sum of probabilities $p_{s}$ over elements $s \in S(b, \delta)$ that are mapped by $\rho_{\ell, \ell^{\prime}}$ to any given $\left(s^{\prime}, t^{\prime}\right) \in S_{\ell}\left(b_{\ell}, \delta\right) \times S_{\ell^{\prime}}\left(b_{\ell^{\prime}}, \delta\right)$ is not too small. Precisely, we assume throughout the paper that for any given $L \geqslant 1$, every $\ell, \ell^{\prime} \in \Lambda \cap[1, L]$ and every $\left(s^{\prime}, t^{\prime}\right) \in S_{\ell}\left(b_{\ell}, \delta\right) \times S_{\ell^{\prime}}\left(b_{\ell^{\prime}}, \delta\right)$,

$$
\sum_{\substack{s \in S(b, \delta) \\ \rho_{\ell, \ell^{\prime}}(s)=\left(s^{\prime}, t^{\prime}\right)}} p_{s} \geqslant \frac{1}{\kappa\left(b_{L}, L, \delta\right)},
$$

which seems an intuitive generalisation of the uniform distribution to a general set $\Lambda$.

By studying the properties of the random walk $\left(X_{k}\right)_{k}$ our aim is to describe the behavior of a "generic" element $g \in G$. To do so, we make use of Kowalski's abstract large sieve procedure extensively described, together with applications, in his book [8]. As in every sieve method, one can only handle cases where the typical properties at issue can be detected locally. To be more precise, we fix for each $\ell \in \Lambda$ a conjugacy invariant subset $\Theta_{\ell} \subset G / H_{\ell}$. The probability we want to upper bound is

$$
\boldsymbol{P}\left(\forall \ell \in \Lambda, \rho_{\ell}\left(X_{k}\right) \notin \Theta_{\ell}\right) \text {. }
$$

When applicable, the method shall produce effective upper bounds for the probability with which $X_{k}$ satisfies a fixed property that can be detected by the condition $\rho_{\ell}\left(X_{k}\right) \notin \Theta_{\ell}$ for some $\Theta_{\ell} \subset G / H_{\ell}$. Our main result is the following abstract sieve statement. We refer the reader to the book by Kowalski [8, Prop. 3.5] for a (self-contained) sieve statement that Theorem 1.1 builds on. For more information on the random walk sieve used here, see also [8, Chap. 7].

Theorem 1.1. With notation as above, we set $\kappa_{N}:=\kappa\left(b_{N}, N, \delta\right)$ for $N \in \mathbf{N}$ and

$$
C_{0}:=\sup _{L \in \mathbf{N}_{>0}} \max _{\substack{\ell \neq \ell^{\prime} \in \Lambda \\ L \leqslant \ell, \ell^{\prime} \leqslant 2 L}} \frac{\# S_{\ell}\left(b_{\ell}, \delta\right)}{\# S_{\ell^{\prime}}\left(b_{\ell^{\prime}}, \delta\right)} .
$$

Assume that condition $(\star)$ holds. Then there exists a positive real $\nu$ such that for every positive integer $k$,

$$
\begin{aligned}
\boldsymbol{P}\left(\rho_{\ell}\left(X_{k}\right) \notin \Theta_{\ell}, \forall \ell \in \Lambda_{L}\right) & \leqslant \boldsymbol{P}\left(C_{0}=\infty\right)+\sum_{\ell \in \Lambda_{L}} e^{-b_{\ell}} \\
& +\left(1+\left(\sum_{\ell \in \Lambda_{L}} n_{\ell}\right)\left(1-\kappa_{2 L}^{-2} \nu\right)^{k}\right)\left(\sum_{\ell \in \Lambda_{L}} \frac{\# \Theta_{\ell}}{n_{\ell}}\right)^{-1},
\end{aligned}
$$

where $L$ is any fixed positive integer $\Lambda_{L}:=\Lambda \cap[L, 2 L]$ and the constant $\nu$ depends only on $C_{0}$, the set $S(b, \delta)$, and the distribution of the steps $\xi_{j}$ (that $i s$, the sequence $\left(p_{s}\right)$ ).

In applications the probability that $C_{0}$ is infinite will be very small via a suitable choice of parameters. Later on we prove a lemma (Lemma 1.5) the purpose of which is to bound efficiently $\boldsymbol{P}\left(C_{0}=\infty\right)$. We end this section by proving the aforementioned uniqueness of admissible sequences, when they exist.

Lemma 1.2. Let $\left(H_{\ell}\right)_{\ell \in \Lambda}$ be a family of subgroups of finite index of $G$. There exists at most one family $\left(R_{\ell}\right)_{\ell \in \Lambda}$ where $R_{\ell}$ is a set of representatives of $G / H_{\ell}$ such that $\left(H_{\ell}, R_{\ell}\right)$ is an admissible local sequence.

Proof. Let $\left(H_{\ell}, R_{\ell}^{(1)}\right)$ and $\left(H_{\ell}, R_{\ell}^{(2)}\right)$ be two admissible local sequences for $G$. Fix $\ell_{0} \in \Lambda$, and let $r_{1} \in R_{\ell_{0}}^{(1)}$ and $r_{2} \in R_{\ell_{0}}^{(2)}$ be representatives of the same element of $G / H_{\ell_{0}}$. So there exists $h \in H_{\ell_{0}}$ such that $r_{1}=r_{2} h$. For any $\ell \neq \ell_{0}$, applying the reduction morphism $\rho_{\ell}$ to the 
above equality yields that $\rho_{\ell}(h)=1$, because of condition (ii) of Definition 1 . Thus $h \in H_{\ell^{\prime}}$ for every $\ell^{\prime} \in \Lambda$. Now, condition (i) of Definition 1 implies that $h=1$, hence $r_{1}=r_{2}$. This shows that $R_{\ell_{0}}^{(1)}=R_{\ell_{0}}^{(2)}$, thereby concluding the proof.

1.2. Cayley graphs on quotients and expansion. Let $G$ be an Abelian ${ }^{1}$ group. We are interested in the properties of the Cayley graphs on the groups $\left(G / H_{\ell}\right)_{\ell \in \Lambda}$ with edges corresponding to the values taken by the random variables $s_{i}^{(\ell)}$ for $i \in\left\{1, \ldots, \kappa\left(b_{\ell}, \ell ; \delta\right)\right\}$. These graphs are regular: the regularity equals the number of distinct values taken by the random variables $s_{i}$.

Throughout the paper, if $\mathcal{G}$ is a $k$-regular graph, then the eigenvalues of $\mathcal{G}$ are the eigenvalues of the normalized adjacency operator $k^{-1} \operatorname{Adj}(\mathcal{G})$. An eigenvalue $\lambda$ is non trivial if $|\lambda| \neq 1$. The spectral gap $\varepsilon(\mathcal{G})$ of $\mathcal{G}$ is defined to be $\min \{1-|\lambda|: \lambda$ is a non trivial eigenvalue of $\mathcal{G}\}$ (recall that the eigenvalue -1 occurs if and only if $\mathcal{G}$ is bipartite). We adopt the following definition for an expander graph, which slightly differs from the standard one. In particular, for us, a $k$-regular graph with spectral gap greater than $1 / 2$ is a $\gamma$-expander graph for any $\gamma \in(0,1 / 2]$.

Definition 2. Let $\gamma$ be a real number satisfying $0<\gamma \leqslant 1 / 2$. A $k$-regular graph $\mathcal{G}$ is a $\gamma$-expander graph if the spectral gap of $\mathcal{G}$ is at least $\gamma$.

The reason for introducing the above setup is a theorem of Alon \& Roichman [3, Th. 1], which has been subsequently improved by Landau \& Russell [9, Th. 2] and Loh \& Schulman [10, Th. 1]. The last improvement obtained so far, which is the version we state and use, is due to Christofides \& Markström [5, Th. 5].

Theorem 1.3 (Christofides-Markström). With notation as above, fix an index $\ell$ in $\Lambda$. For every $\delta \in(0,1 / 2]$, the probability that $X\left(G / H_{\ell},\left\{s_{1}^{(\ell)}, \ldots, s_{\kappa\left(b_{\ell}, \ell ; \delta\right)}^{(\ell)}\right\}\right)$ is not a $\delta$-expander graph is less than $e^{-b_{\ell}}$

The statement can be rephrased by saying it is highly probable that the Cayley graph $X\left(G / H_{\ell},\left\{s_{1}^{(\ell)}, \ldots, s_{\kappa\left(b_{\ell}, \ell ; \delta\right)}^{(\ell)}\right\}\right)$ be a $\delta$-expander graph, the counterpart being that the edge set has very large cardinality. Note that the definition of an expander graph we use is not completely equivalent to the usual definition. However, it is a standard fact that the (usual) expansion property and the spectral gap property are closely related notions (see, e.g., [6, Th. 1.2.3]), which allows us to use our definition harmlessly for our purposes.

Kowalski [8, Chap. 7] successfully combines large sieve techniques with expansion properties in the setting of random walks on arithmetic groups. We want to transpose this principle in a combinatorial setting. When adapting Kowalski's work a non trivial issue comes from the fact that the expansion property crucial to us is not automatically stable under Cartesian product. Precisely, loc. cit. relies on the fact that if $S$ is a symmetric generating system for $\mathrm{SL}_{2}(\mathbf{Z})$ and if $\pi_{d}: \mathrm{SL}_{2}(\mathbf{Z}) \rightarrow \mathrm{SL}_{2}(\mathbf{Z} / d \mathbf{Z})$ is the reduction modulo $d$ map for some $d \geqslant 2$, then the whole family of Cayley graphs on $\mathrm{SL}_{2}(\mathbf{Z}) / \operatorname{ker} \pi_{d}$ (with respect to the projection of $S$ ) indexed by squarefree integers is expanding. In fact it would be enough to have the same result with an index set replaced by the set of positive integers that are squarefree and products of at most two primes. (However, for Kowalski's purposes, considering the primes as the index set would not be sufficient.) To obtain a suitable combinatorial analogue of this method, the forthcoming lemma is sufficient. It shows that expansion properties of Cayley graphs are preserved, albeit only imperfectly, when one takes the Cartesian product of two base groups. The expansion ratio guaranteed by the lemma is strong enough for our purposes. However we refer the interested reader to [1] for a

\footnotetext{
${ }^{1}$ The assumption that $G$ is Abelian is unnecessary for most of the results of this section, but our main result and the applications we develop only involve Abelian groups, so we stick to this case where the exposition is simpler.
} 
much more sophisticated method that does produce expander "product Cayley graphs". (Recall that the definition of "expander graph" we use slightly differs from the standard one.)

Lemma 1.4. Let $\delta \in(0,1 / 2]$. With notation as above assume that $X(G, S)$ and $X(H, T)$ are $\delta$-expander Cayley graphs on finite Abelian groups $G$ and $H$ (with edge set defined by $S \subseteq G$ and $T \subseteq H$, respectively). Then for every $\left(x_{0}, y_{0}\right) \in G \times H$ with $x_{0}^{2}=1=y_{0}^{2}$, the Cayley graph $X\left(G \times H,\left(S \times\left\{y_{0}\right\}\right) \cup\left(\left\{x_{0}\right\} \times T\right)\right)$ is a $\left((1+\gamma)^{-1} \delta\right)$-expander graph, where

$$
\gamma:=\max \left\{\frac{\left|S \cup S^{-1}\right|}{\left|T \cup T^{-1}\right|}, \frac{\left|T \cup T^{-1}\right|}{\left|S \cup S^{-1}\right|}\right\} .
$$

Proof. For convenience, set $Y:=\left(S \times\left\{y_{0}\right\}\right) \cup\left(\left\{x_{0}\right\} \times T\right), S^{*}:=S \cup S^{-1}, T^{*}:=T \cup T^{-1}$ and $Y^{*}:=Y \cup Y^{-1}$. The eigenfunctions of the normalized adjacency operator on $X(G \times H, Y)$ are of the form

$$
(\chi, \tau):(g, h) \mapsto \chi(g) \tau(h),
$$

for characters $\chi \in \hat{G}$ and $\tau \in \hat{H}$. The corresponding eigenvalues are of the form

$$
\lambda_{\chi, \tau}:=\frac{1}{\left|S^{*}\right|+\left|T^{*}\right|} \sum_{(g, h) \in Y^{*}} \chi(g) \tau(h) .
$$

Since $x_{0}^{2}=1=y_{0}^{2}$ the sum splits as follows:

$$
\left(\left|S^{*}\right|+\left|T^{*}\right|\right) \lambda_{\chi, \tau}=\tau\left(y_{0}\right) \sum_{g \in S^{*}} \chi(g)+\chi\left(x_{0}\right) \sum_{h \in T^{*}} \tau(h) .
$$

We deduce that

$$
\left|\lambda_{\chi, \tau}\right| \leqslant \frac{\left|S^{*}\right|}{\left|S^{*}\right|+\left|T^{*}\right|}\left|\frac{1}{\left|S^{*}\right|} \sum_{g \in S^{*}} \chi(g)\right|+\frac{\left|T^{*}\right|}{\left|S^{*}\right|+\left|T^{*}\right|}\left|\frac{1}{\left|T^{*}\right|} \sum_{h \in T^{*}} \tau(h)\right| .
$$

If both $\chi$ and $\tau$ are non-trivial, then $\left|\lambda_{\chi, \tau}\right| \leqslant 1-\delta$ since each of $X(G, S)$ and $X(H, T)$ are $\delta$-expanders where $\delta \in(0,1 / 2]$. If $\chi$ is trivial and $\tau$ is non trivial, we obtain instead

$$
\left|\lambda_{\chi, \tau}\right| \leqslant 1-\delta\left(1+\left|S^{*}\right| /\left|T^{*}\right|\right)^{-1},
$$

hence the result by symmetry of the roles played by $G$ and $H$.

To better comprehend Lemma 1.4, we give several examples, which also allow us to demonstrate the necessity of its hypothesis and the optimality of the bound given. For a positive integer $n$, we let $\mathbf{Z}_{n}$ be the cyclic group of order $n$. Consider first the case where both $G$ and $H$ are $\mathbf{Z}_{4}$, with $S$ and $T$ each consisting of a generating element of $\mathbf{Z}_{4}$. Thus the graphs $X(G, S)$ and $X(G, T)$ are isomorphic to the undirected cycle $C_{4}$ with 4 vertices. (Recall that $X(G, S)=X\left(G, S^{*}\right)$ by the definition.) The spectral gap of $C_{4}$ is 1 . Now choose $x_{0}$ and $y_{0}$ to be the neutral elements of $G$ and $H$, respectively. The hypothesis of Lemma 1.4 are thus satisfied. Note that $\gamma=1$, so according to this lemma, the graph $X:=X\left(G \times H,\left(\left\{x_{0}\right\} \times T\right) \cup\left(S \times\left\{y_{0}\right\}\right)\right.$ should have spectral gap at least $(1+1)^{-1} \cdot 1=\frac{1}{2}$. To check this, observe that $X$ is the 4-regular graph depicted in Figure 1: it consists of two disjoint cycles of size 8 the vertices of which are "linked using cycles of length 4". This graph indeed has spectral gap exactly $\frac{1}{2}$. This can actually be directly deduced from the proof of Lemma 1.4 by using (3), thereby obtaining a precise expression for the eigenvalues of the product graph. More generally, one deduces that performing the same construction as we just did but starting from $\mathbf{Z}_{2 k}$ for any integer $k \geqslant 2$ yields an infinite family of examples where the bound given by Lemma 1.4 is attained, showing its optimality. (The spectral gap of the two (isomorphic) starting graphs will be $1-|\cos (\pi(k+1) / k)|$ and that of the product graph exactly half this quantity.) 
The hypothesis that $x_{0}$ and $y_{0}$ must be elements with order at most 2 in their respective groups is necessary, as is seen by taking $G:=\mathbf{Z}_{3} \times \mathbf{Z}_{5}=\langle\sigma\rangle \times\langle\tau\rangle$ and $H$ the dihedral group of order 6 . Letting $\mu$ be a generator of $H$, we set $S:=\{\sigma\}, T:=\{\mu\}, x_{0}:=\sigma \tau$ and $y_{0}:=\pi^{3}$. (Thus $y_{0}$ is of order 2 while $x_{0}$ is of order 15.) The graph $X(G, S)$ consists of five disjoint triangles while $X(H, T)$ consists of two disjoint cycles of length 6 . Consequently each of these graphs has spectral gap $\frac{1}{2}$. However, the spectral gap of the graph $X\left(G \times H,\left(\left(\left\{x_{0}\right\} \times T\right) \cup\left(S \times\left\{y_{0}\right\}\right)\right)\right)$ is less than 0.045 , which is less than $\frac{1}{2} \cdot(1+\gamma)^{-1}=\frac{1}{4}$.

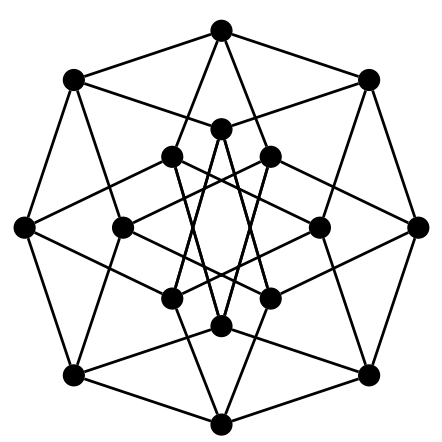

FiguRE 1. The product graph $X\left(\mathbf{Z}_{4} \times \mathbf{Z}_{4},\{(1, \sigma),(\sigma, 1)\}\right)$, where $\sigma$ is a generating element of $\mathbf{Z}_{4}$.

In applications, it is important to keep control of the "spectral gap loss", that is, the size of the parameter $\gamma$ appearing in Lemma 1.4. To do so we need the following technical lemma, which asserts in a precise quantitative way that it is harmless to suppose that the random variables $s_{i}^{(\ell)}$ take distinct values when evaluated simultaneously and as long as $n_{\ell}$ is fairly larger than $\kappa\left(b_{\ell}, \ell ; \delta\right)$. This is a simple application of standard concentration principles.

Lemma 1.5. Keeping notation as above, fix $\ell \in \Lambda$. For readability, $\kappa\left(b_{\ell}, \ell ; \delta\right)$ is abbreviated to $\kappa_{\ell}$. Let $X$ be the random variable that counts the number of distinct values in the multiset $\left\{s_{1}^{(\ell)}, \ldots, s_{\kappa_{\ell}}^{(\ell)}\right\}$. One has:

(a) if there exists a positive real $\varepsilon$, independent of $\ell$, such that $1-\kappa_{\ell} / n_{\ell}>\varepsilon$, then

$$
\boldsymbol{P}\left(X<\kappa_{\ell} / 2\right) \leqslant 2 \exp \left(-\kappa_{\ell} \varepsilon^{2} / 8\right)
$$

(b) if $n_{\ell} \leqslant \kappa_{\ell}$, then

$$
\boldsymbol{P}\left(X<n_{\ell} / 2\right) \leqslant\left(\begin{array}{c}
n_{\ell} \\
\left\lceil n_{\ell} / 2\right\rceil
\end{array}\right) \cdot 2^{-\kappa_{\ell}}
$$

Proof. Let $x_{1}, \ldots, x_{n_{\ell}}$ be the elements of $G / H_{\ell}$. For each $i \in\left[n_{\ell}\right]$, let $X_{i}$ be the 0 - 1 random variable that is equal to 1 if $x_{i} \in\left\{s_{j}^{\ell}: 1 \leqslant j \leqslant n_{\ell}\right\}$. Notice that $X=\sum_{i=1}^{n_{\ell}} X_{i}$. Consequently, the linearity of expectation implies that $\boldsymbol{E}(X)=\sum_{i=1}^{n_{\ell}} \boldsymbol{E}\left(X_{i}\right)$. Moreover, for each $i \in\left[n_{\ell}\right]$,

$$
\boldsymbol{E}\left(X_{i}\right)=1-\boldsymbol{P}\left(X_{i}=0\right)=1-\left(1-\frac{1}{n_{\ell}}\right)^{\kappa_{\ell}} \geqslant 1-\exp \left(-\kappa_{\ell} / n_{\ell}\right) \geqslant \kappa_{\ell} / n_{\ell}-1 / 2 \cdot\left(\kappa_{\ell} / n_{\ell}\right)^{2}
$$

so that $\boldsymbol{E}(X) \geqslant \kappa_{\ell}-1 / 2 \cdot \kappa_{\ell}^{2} / n_{\ell}$.

Now, since $X$ is determined by $\kappa_{\ell}$ independent trials and, for every possible outcome of the trials changing the outcome of any one trial can affect $X$ by at most 1 , the Simple Concentration 
Bound [13, p. 79] yields that for every positive number $t$,

$$
\boldsymbol{P}(|X-\boldsymbol{E}(X)|>t) \leqslant 2 \exp \left(-\frac{t^{2}}{2 \kappa_{\ell}}\right) .
$$

Therefore, to prove $((\mathrm{a}))$ one sets $t:=\kappa_{\ell} / 2 \cdot\left(1-\kappa_{\ell} / n_{\ell}\right)$. This implies that

$$
\boldsymbol{P}\left(X<\kappa_{\ell} / 2\right) \leqslant 2 \exp \left(-\frac{\kappa_{\ell}}{8}\left(1-\kappa_{\ell} / n_{\ell}\right)^{2}\right) \leqslant 2 \exp \left(-\frac{\kappa_{\ell} \varepsilon^{2}}{8}\right) .
$$

To prove $((\mathrm{b}))$ one rather proceeds as follows. Notice that $X<n_{\ell} / 2$ if and only if there exists a subset $H^{\prime}$ of $G / H_{\ell}$ of size $\left\lceil n_{\ell} / 2\right\rceil$ such that $\left\{s_{i}^{\ell}: 1 \leqslant i \leqslant \kappa_{\ell}\right\} \cap H^{\prime}=\varnothing$. This happens with probability at most $2^{-\kappa_{\ell}}$. Consequently, we infer that

$$
\boldsymbol{P}\left(X<n_{\ell}\right) \leqslant\left(\begin{array}{c}
n_{\ell} \\
\left\lceil n_{\ell} / 2\right\rceil
\end{array}\right) \cdot 2^{-\kappa_{\ell}} .
$$

1.3. Random walk large sieve: proof of the main result. We first state an easy consequence of the definition of $S(b, \delta)$ that is useful in our sieving procedure.

Lemma 1.6. For all distinct integers $\ell, \ell^{\prime} \in \Lambda$, one has

$$
\rho_{\ell}(S(b ; \delta))=S_{\ell}\left(b_{\ell}, \delta\right) \cup\{1\} \quad \text { and } \quad \rho_{\ell, \ell^{\prime}}(S(b, \delta))=\left(S_{\ell}\left(b_{\ell}, \delta\right) \cup\{1\}\right) \times\left(S_{\ell^{\prime}}\left(b_{\ell^{\prime}}, \delta\right) \cup\{1\}\right) .
$$

Proof. We use condition (ii) in Definition 1: the image by $\rho_{\ell}$ of $S(b, \delta)$ is the product of elements all equal to 1 except maybe for the $\ell$-factor which can be any element of $\rho_{\ell}\left(\tilde{S}_{\ell}\left(b_{\ell}, \delta\right) \cup\{1\}\right)$, that is, any element of $S_{\ell}\left(b_{\ell}, \delta\right) \cup\{1\}$.

The second equality is obtained using the same argument.

We now define one last piece of useful notation before starting the proof of Theorem 1.1. For indices $\ell$ and $\ell^{\prime}$ in $\Lambda$, we set $G_{\ell, \ell^{\prime}}:=G / H_{\ell} \times G / H_{\ell}^{\prime}$ if $\ell \neq \ell^{\prime}$ and $G_{\ell}\left(=G_{\ell, \ell^{\prime}}\right):=G / H_{\ell}$ otherwise. The proof of Theorem 1.1 is based on an adaptation of that of [8, Prop. 7.2].

Proof of Theorem 1.1. Fix a real number $\delta$ in $(0,1 / 2]$ and let us split the probability we are interested in:

$$
\begin{aligned}
& \boldsymbol{P}\left(\forall \ell \in \Lambda_{L}, \rho_{\ell}\left(X_{k}\right) \notin \Theta_{\ell}\right) \leqslant \boldsymbol{P}\left(C_{0}=\infty\right) \\
& +\boldsymbol{P}\left(\exists \ell \in \Lambda_{L}, X\left(G / H_{\ell}, \rho_{\ell}(S(b, \delta))\right) \text { is not a } \delta \text {-expander }\right) \\
& +\boldsymbol{P}\left(\left(C_{0}<\infty\right) \wedge\left(\forall \ell \in \Lambda_{L}, X\left(G / H_{\ell}, \rho_{\ell}(S(b, \delta)) \text { is a } \delta \text {-expander and } \rho_{\ell}\left(X_{k}\right) \notin \Theta_{\ell}\right)\right) .\right.
\end{aligned}
$$

As we shall see, the third summand can be bounded from above using sieving techniques. Moreover, the second summand can be handled by invoking Theorem 1.3. Indeed, since $\rho_{\ell}(S(b, \delta))=S_{\ell}\left(b_{\ell}, \delta\right) \cup\{1\}$ by Lemma 1.6, we can show that the following statement holds (note that the statement would be trivial if we were only interested in edge-expansion):

$$
\begin{aligned}
& \boldsymbol{P}\left(\exists \ell \in \Lambda_{L}, X\left(G / H_{\ell}, \rho_{\ell}(S(b, \delta))\right) \text { is not a } \delta \text {-expander }\right) \\
& \quad \leqslant \boldsymbol{P}\left(\exists \ell \in \Lambda_{L}, X\left(G / H_{\ell}, S_{\ell}\left(b_{\ell}, \delta\right)\right) \text { is not a } \delta \text {-expander }\right) .
\end{aligned}
$$

This inequality is a consequence of Lemma 1.7 that we state and prove at the end of this section. Applying Theorem 1.3 yields that

$$
\boldsymbol{P}\left(\exists \ell \in \Lambda_{L}, X\left(G / H_{\ell}, S_{\ell}\left(b_{\ell}, \delta\right)\right) \text { is not a } \delta \text {-expander }\right) \leqslant \sum_{\ell \in \Lambda_{L}} e^{-b_{\ell}} .
$$


Let us now turn to the third summand of the right side of (4). First, notice that

$$
\begin{aligned}
& \boldsymbol{P}\left(\left(C_{0}<\infty\right) \wedge\left(\forall \ell \in \Lambda_{L}, X\left(G / H_{\ell}, \rho_{\ell}(S(b, \delta))\right) \text { is a } \delta \text {-expander and } \rho_{\ell}\left(X_{k}\right) \notin \Theta_{\ell}\right)\right) \\
\leqslant & \boldsymbol{P}\left(\forall \ell \in \Lambda_{L}, \rho_{\ell}\left(X_{k}\right) \notin \Theta_{\ell} \mid C_{0}<\infty \text { and } \forall \ell \in \Lambda_{L}, X\left(G / H_{\ell}, \rho_{\ell}(S(b, \delta))\right) \text { is a } \delta \text {-expander }\right) .
\end{aligned}
$$

Now we are in a situation close to the axiomatic sieve method developed in [8]. We fix (non-necessarily distinct) indices $\ell$ and $\ell^{\prime}$ in $\Lambda_{L}$ (defining $\rho_{\ell, \ell}$ to be $\rho_{\ell}$ ) and a character $\lambda$ of $G_{\ell, \ell^{\prime}}$

$$
\lambda: G \stackrel{\rho_{\ell, \ell^{\prime}}}{\rightarrow} G_{\ell, \ell^{\prime}} \stackrel{\lambda_{0}}{\rightarrow} \mathbf{C}^{\times},
$$

factoring through $G_{\ell, \ell^{\prime}}$ in such a way that $\lambda_{0}$ is a non trivial character of $G_{\ell, \ell^{\prime}}$.

We first prove the following statement. Assume that $C_{0}<\infty$ and $X\left(G / H_{\ell}, \rho_{\ell}(S(b, \delta))\right)$ is a $\delta$-expander for every $\ell \in \Lambda$. We assert that there exists a positive constant $\nu$ depending only on $C_{0}$, the set $S(b, \delta)$ and the distribution of the steps $\xi_{j}$ (that is, the sequence $\left(p_{s}\right)$ ), such that

$$
\left|\boldsymbol{E}\left(\lambda\left(X_{k}\right)\right)\right| \leqslant\left(1-\kappa_{2 L}^{-2} \nu\right)^{k} .
$$

Consider:

$$
M:=\boldsymbol{E}\left(\lambda\left(\xi_{k}\right)\right)=\sum_{s \in S(b, \delta)} p(s) \lambda(s),
$$

which is a well-defined element of $\mathbf{C}^{\times}$since the series defining $M$ converges absolutely. Let us also consider the complex number $M^{+}:=1-M$.

Note that $M$ and $M^{+}$are in fact real numbers since the set $S(b, \delta)$ as well as the distribution of the steps $\xi_{k}$ are symmetric. We also need to define

$$
N_{0}:=\boldsymbol{E}\left(\lambda\left(X_{0}\right)\right)=\sum_{t \in T} \boldsymbol{P}\left(X_{0}=t\right) \lambda(t) \in \mathbf{C}^{\times},
$$

where $T$ is a fixed (finite) subset of $G$ containing the starting point $g_{0}$ of the random walk $\left(X_{k}\right)$. (For simplicity one can assume that $T=\left\{g_{0}\right\}$.)

The random variables $X_{0}$ and $\xi_{k}$ being independent, it follows that for every positive integer $k$,

$$
\boldsymbol{E}\left(\lambda\left(X_{k}\right)\right)=N_{0} M^{k} .
$$

We have $\left|N_{0}\right| \leqslant 1$ and we compute

$$
\begin{aligned}
M^{+} & =\sum_{s \in S(b, \delta)} p_{s}(1-\lambda(s))=\sum_{\left.\left(s^{\prime}, t^{\prime}\right) \in S_{\ell}\left(b_{\ell}, \delta\right) \times S_{\ell^{\prime}}\left(b_{\ell^{\prime}}, \delta\right)\right)}\left(\sum_{\substack{s \in S(b, \delta) \\
\rho_{\ell, \ell^{\prime}}(s)=\left(s^{\prime}, t^{\prime}\right)}} p_{s}\right)\left(1-\lambda_{0}\left(s^{\prime}, t^{\prime}\right)\right) \\
& \geqslant \frac{1}{\kappa\left(b_{2 L}, 2 L, \delta\right)^{2}} \min _{\psi \neq 1} \max _{\left.\left(s^{\prime}, t^{\prime}\right) \in S_{\ell}\left(b_{\ell}, \delta\right) \times S_{\ell^{\prime}}\left(b_{\ell^{\prime}}, \delta\right)\right)}\left(1-\psi\left(s^{\prime}, t^{\prime}\right)\right)
\end{aligned}
$$

where $\psi$ runs over the non trivial characters of $G_{\ell, \ell^{\prime}}$. With the same notation we deduce that for any $a \in S_{\ell}\left(b_{\ell}, \delta\right)$ and any $b \in S_{\ell^{\prime}}\left(b_{\ell^{\prime}}, \delta\right)$ both of order at most 2 ,

$$
M^{+} \geqslant \kappa\left(b_{2 L}, 2 L, \delta\right)^{-2} \min _{\psi \neq 1} \max _{\left.\left(s^{\prime}, t^{\prime}\right) \in S_{\ell}\left(b_{\ell}, \delta\right) \times\{b\} \cup\{a\} \times S_{\ell^{\prime}}\left(b_{\ell^{\prime}}, \delta\right)\right)}\left(1-\psi\left(s^{\prime}, t^{\prime}\right)\right) .
$$

Lemma 1.4 asserts that the family of Cayley graphs with vertex set $G_{\ell, \ell^{\prime}}$ and edge set $S_{\ell}\left(b_{\ell}, \delta\right) \times$ $\left.\{b\} \cup\{a\} \times S_{\ell^{\prime}}\left(b_{\ell^{\prime}}, \delta\right)\right)$ is a family of $\left(1+C_{0}\right)^{-1} \delta$-expanders as soon as the family $\left(X\left(G_{\ell}, S_{\ell}\left(b_{\ell}, \delta\right)\right)\right)_{\ell \in \Lambda}$ is a family of $\delta$-expanders. Thus we can appeal to the translation of Lubotzky's property $(\tau)$ into the property of expansion of the corresponding Cayley graphs (see [12, Prop. 2.5]) to justify the existence of a positive constant $\nu\left(C_{0}, S(b, \delta),\left(p_{s}\right)\right)$ which is uniform in $\ell, \ell^{\prime} \in \Lambda$ and such that

$$
M^{+} \geqslant \kappa\left(b_{2 L}, 2 L, \delta\right)^{-2} \nu\left(C_{0}, S(b, \delta),\left(p_{s}\right)\right) \text {. }
$$


To conclude the proof of the claim it suffices to observe that, because of our definition of expansion, the fact that the family $X\left(G_{\ell}, \rho_{\ell}(S(b, \delta))\right)$ is assumed to be a family of $\delta$-expanders implies that these Cayley graphs are not bipartite and hence producing a lower bound for $1+M$ is not required.

We can now finish the proof by using Kowalski's large sieve inequality [8, Prop. 3.7]. We obtain $\boldsymbol{P}\left(\forall \ell \in \Lambda_{L}, \rho_{\ell}\left(X_{k}\right) \notin \Theta_{\ell} \mid C_{0}<\infty\right.$ and $\forall \ell \in \Lambda_{L} X\left(G / H_{\ell}, \rho_{\ell}(S(b, \delta))\right)$ is a $\delta$-expander $)$

$$
\leqslant \Delta\left(X_{k} ; L\right)\left(\sum_{\ell \in \Lambda_{L}} \frac{\# \Theta_{\ell}}{n_{\ell}}\right)^{-1},
$$

where one has the theoretical upper bound:

with

$$
\Delta\left(X_{k} ; L\right) \leqslant \max _{\ell \in \Lambda_{L}} \max _{\chi \in \mathcal{B}_{\ell}^{*}} \sum_{\ell^{\prime} \in \Lambda_{L}} \sum_{\chi^{\prime} \in \mathcal{B}_{\ell^{\prime}}^{*}}\left|W\left(\chi, \chi^{\prime}\right)\right|,
$$

$$
W\left(\chi, \chi^{\prime}\right):=\boldsymbol{E}\left(\chi \rho_{\ell}\left(X_{k}\right) \overline{\chi^{\prime} \rho_{\ell^{\prime}}\left(X_{k}\right)}\right)=\boldsymbol{E}\left(\left[\chi, \overline{\chi^{\prime}}\right] \rho_{\ell, \ell^{\prime}}\left(X_{k}\right)\right) .
$$

Here for any $\ell \in \Lambda$ we let $\mathcal{B}_{\ell}$ be the character group of $G / H_{\ell}$. We set further $\mathcal{B}_{\ell}^{*}:=\mathcal{B}_{\ell} \backslash\{1\}$. Finally if $\psi_{i}$ is a character of a finite Abelian group $G_{i}$ for $i \in\{1,2\}$, then we let $\left[\psi_{1}, \psi_{2}\right]$ be the character $\pi \otimes \tau$ of $G \times H$ if $G \neq H$ or of $G$ otherwise.

In our setting, using [8, Lemma 3.4] we deduce that

$$
\left[\chi, \overline{\chi^{\prime}}\right] \rho_{\ell, \ell^{\prime}}=\delta\left((\ell, \pi),\left(\ell^{\prime}, \tau\right)\right) \mathbf{1}+\left[\chi \overline{\chi^{\prime}}\right]_{0} \rho_{\ell, \ell^{\prime}},
$$

where $\delta(\cdot, \cdot)$ is the Kronecker symbol and $\left[\chi, \overline{\chi^{\prime}}\right]_{0}$ is the component of $\left[\chi, \overline{\chi^{\prime}}\right]$ orthogonal to the trivial character $\mathbf{1}$. Thus applying (5) to

$$
\lambda:=\left[\chi, \overline{\chi^{\prime}}\right]_{0} \rho_{\ell, \ell^{\prime}}
$$

we obtain

$$
\left|\boldsymbol{E}\left(\left[\chi, \overline{\chi^{\prime}}\right]_{0} \rho_{\ell, \ell^{\prime}}\left(X_{k}\right)\right)\right| \leqslant\left(1-\nu \kappa_{2 L}^{-2}\right)^{k} .
$$

Putting everything together we deduce as wished that

$$
\begin{aligned}
& \boldsymbol{P}\left(\forall \ell \in \Lambda_{L}, \rho_{\ell}\left(X_{k}\right) \notin \Theta_{\ell} \mid C_{0}<\infty \text { and } \forall \ell \in \Lambda_{L} X\left(G / H_{\ell}, \rho_{\ell}(S(b, \delta))\right) \text { is a } \delta \text {-expander }\right) \\
\leqslant & \left(1+\left(\sum_{\ell \in \Lambda_{L}} n_{\ell}\right)\left(1-\nu \kappa_{2 L}^{-2}\right)^{k}\right)\left(\sum_{\ell \in \Lambda_{L}} \frac{\# \Theta_{\ell}}{n_{\ell}}\right)^{-1} .
\end{aligned}
$$

It remains to prove the following statement.

Lemma 1.7. Let $G_{0}$ be an Abelian group and let $S$ be a subset of $G_{0}$. If $X\left(G_{0}, S\right)$ is a $\delta$-expander graph, then so is $X\left(G_{0}, S \cup\{1\}\right)$.

Proof. The statement is trivially true if $1 \in S$, so we assume that $1 \notin S$. Set $S^{*}:=S \cup S^{-1}$ and $s^{*}:=\# S^{*}$. Recall that Definition 2 implies that $\delta \in(0,1 / 2]$. To prove the statement, it suffices to show that every non trivial eigenvalue $\lambda^{\prime}$ of $X\left(G_{0}, S \cup\{1\}\right)$ is such that $\left|\lambda^{\prime}\right| \leqslant 1 / 2$ or $\left|\lambda^{\prime}\right| \leqslant|\lambda|$ for some non trivial eigenvalue $\lambda$ of $X\left(G_{0}, S\right)$.

Let $\lambda^{\prime}$ be a non trivial eigenvalue of $X\left(G_{0}, S \cup\{1\}\right)$. Using the usual convention according to which a loop contributes 2 to the degree of a vertex, we deduce that $\lambda^{\prime}=\left(2+s^{*}\right)^{-1}\left(\sum_{s \in S^{*}} \chi(s)+\right.$ $\chi(1))$ for some non trivial character $\chi$ of $G_{0}$. Therefore,

$$
\lambda^{\prime}=\frac{s^{*}}{2+s^{*}} \lambda+\frac{1}{2+s^{*}}=\lambda+\frac{1-2 \lambda}{2+s^{*}},
$$


where $\lambda:=\left(s^{*}\right)^{-1} \sum_{s \in S^{*}} \chi(s)$ is a non trivial eigenvalue of $X\left(G_{0}, S\right)$.

Consequently, it is enough to prove that if $\left|\lambda^{\prime}\right|>1 / 2$, then $\left|\lambda^{\prime}\right| \leqslant|\lambda|$. Suppose, on the contrary, that $\left|\lambda^{\prime}\right|>1 / 2$ and $\left|\lambda^{\prime}\right|>|\lambda|$. Then $\lambda^{\prime}>0$. Indeed, otherwise $\lambda \leqslant-1 / s^{*}<0$ and hence $-\lambda+\frac{2 \lambda-1}{2+s^{*}}>|\lambda|=-\lambda$ implies that $\lambda>1 / 2$, a contradiction.

Hence, $\lambda^{\prime}>1 / 2$, which yields that $\lambda>1 / 2$. However, this implies that $\frac{1-2 \lambda}{2+s^{*}}<0$, so that $\lambda>\lambda^{\prime}=\left|\lambda^{\prime}\right|$, contrary to our assumption. This finishes the proof.

\section{Illustrative EXAMPLES}

This section illustrate how Theorem 1.1 can be applied to various classical topics. Let us state two bounds on $\psi$ that are useful in the forthcoming applications. An elementary study of the function $\psi$, which extends continuously to $[0,1 / 2]$, shows that $\psi$ is increasing on that interval and therefore:

$$
1.442 \ldots \simeq(1 / \log 2) \leqslant \psi(\delta) \leqslant 4 / \log (27 / 16) \simeq 7.644 \ldots
$$

for any $\delta \in(0,1 / 2]$. We now present some applications inspired by the classical Ramsey Theory.

2.1. Towards a quantitative infinite Ramsey theory. Our purpose is to illustrate how our method can be applied in the context of infinite Ramsey theory. Let us first recall the result we have in mind, established by Ramsey [14]. Given a set $X$ and a non-negative integer $r$, we define $X^{(r)}$ to be the collection of all subsets of $X$ of cardinality $r$.

Theorem 2.1 (Infinite Ramsey Theorem [14]). Let $X$ be some countably infinite set. Let $c$ and $r$ be positive integers. Consider a given colouring $f: X^{(r)} \rightarrow \mathbf{Z} / c \mathbf{Z}$ of the elements of $X^{(r)}$ in $c$ different colours. Then there exists some infinite subset $A$ of $X$ such that the function $f$ is constant on $A^{(r)}$, that is, all subsets of $A$ of cardinality $r$ have the same image under $f$.

For every function $f$, the support of $f$ is the set of all elements $e$ in the domain of $f$ such that $f(e) \neq 0$. As in the statement of Ramsey's Theorem, fix positive integers $c$ and $r$. As our base set we choose $X:=\mathbf{N}_{\geqslant 1}$. The set $\mathscr{C}^{(r)}$ of all possible $c$-colourings of subsets of cardinality $r$ of $X$ may be endowed with a group structure inherited from that of $\mathbf{Z} / c \mathbf{Z}$. Explicitly, the addition of two elements $f$ and $g$ of $\mathscr{C}^{(r)}$ is formally defined by

$$
f+g: X^{(r)} \rightarrow \mathbf{Z} / c \mathbf{Z}, \quad A \mapsto f(A)+g(A) .
$$

The neutral element is the function that is identically 0 .

We also fix an auxiliary positive integer $j$ and we set $\Lambda:=\mathbf{N}_{\geqslant 1}$. We consider the subsets $I_{\ell}^{(r, j)}:=\{(r+j)(\ell-1)+1, \ldots,(r+j) \ell\}$ of $X$ indexed by $\ell \in \Lambda$. If $j$ and $r$ are fixed, then $I_{\ell}^{(r, j)}$ is an integral interval of size $r+j$ and different indices $\ell$ and $\ell^{\prime}$ give rise to disjoint intervals $I_{\ell}^{(r, j)}$ and $I_{\ell^{\prime}}^{(r, j)}$. For $\ell \in \Lambda$, let $E_{\ell}^{(r)}$ be the set of subsets of size $r$ of $I_{\ell}^{(r, j)}$. Let $C_{\ell}$ be the collection of all colourings supported on $E_{\ell}^{(r)}$ and let $H_{\ell}$ be the subgroup of all colourings of $\mathscr{C}^{(r)}$ supported on the complement of $E_{\ell}^{(r)}$ in $X^{(r)}$. This way $C_{\ell}$ is a set of representatives for the quotient $\mathscr{C}^{(r)} / H_{\ell}$. Indeed, no two distinct functions in $C_{\ell}$ are congruent modulo an element of $H_{\ell}$. Moreover, for any $f \in \mathscr{C}$, let $f_{C}$ be the coloring equal to $f$ on $E_{\ell}^{(r)}$ and equal to 0 everywhere else. It follows that $f_{C} \in C_{\ell}$ and $f-f_{C} \in H_{\ell}$, or equivalently $f \equiv f_{C}\left(\bmod H_{\ell}\right)$. Let $\rho_{\ell}: \mathscr{C}^{(r)} \rightarrow \mathscr{C}^{(r)} / H_{\ell}$ be the canonical surjection. The disjointness of the sets $I_{\ell}^{(r, j)}$ readily implies that $\left(H_{\ell}, C_{\ell}\right)$ is an admissible local sequence for $\mathscr{C}^{(r)}$. In addition, we note that $\left|E_{\ell}^{(r)}\right|=\left(\begin{array}{c}r+j \\ r\end{array}\right)$. Summing-up, we thus established the following statement.

Lemma 2.2. The sequence $\left(H_{\ell}, C_{\ell}\right)$ is an admissible local sequence for $\mathscr{C}^{(r)}$ and

$$
\forall \ell \in \Lambda, \quad n_{\ell}:=\left(\mathscr{C}^{(r)}: H_{\ell}\right)=\# C_{\ell}=c^{\left(\begin{array}{c}
r+j \\
r
\end{array}\right) .}
$$


We may now define on $\mathscr{C}^{(r)}$ a random walk $\left(X_{k}\right)$ that satisfies the requirements of Theorem 1.1. We then ask the question:

at which speed do we reach a colouring $X_{k}$ of the $r$-element subsets of $X$ that exhibits a subset $A \subseteq$ $X$ of size $r+j$, all the $r$-element subsets of which have the same colour?

The next statement answers that question.

Theorem 2.3. Let $\left(X_{k}\right)$ be the random walk defined on $\mathscr{C}^{(r)}$ as in Subsection 1.3, with $\widetilde{S}_{\ell}(b, \delta) \subseteq$ $C_{\ell}$. Fix positive integers $j, r, c$ and a positive real number $\varepsilon$. Then for every positive integer $k$, $\boldsymbol{P}\left(\right.$ No element of $\mathbf{N}^{(j+r)}$ has all its $r$-element subsets of the same colour in $\left.X_{k}\right) \ll k^{-1 / 2+\varepsilon}$, where the implied constant depends only on $\varepsilon, j, r, c, C_{0}, S(b, \delta)$, and the sequence $\left(p_{s}\right)$. This constant could be explicitly computed in terms of $\varepsilon, j, r, c$, and the constant $\nu$ of Theorem 1.1. As a function of $j$, this constant is unbounded.

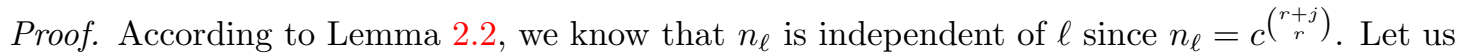
set $b_{\ell}:=\ell$ for all $\lambda \in \Lambda$. Thus $\kappa\left(b_{\ell}, \ell ; \delta\right) \geqslant \psi(\delta)\left(\log n_{\ell}+\ell+\log 2\right)$, where $\psi$ is defined by $(1)$.

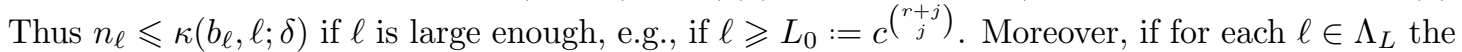
set $S_{\ell}\left(b_{\ell} ; \delta\right)$ contains at least $n_{\ell} / 2$ distinct elements, then $C_{0} \leqslant 2$. Therefore, applying Lemma 1.5 we deduce that for for every $L \geqslant L_{0}$,

$$
\begin{aligned}
\boldsymbol{P}\left(C_{0}=\infty\right) & \leqslant \boldsymbol{P}\left(\exists \ell \in \Lambda_{L}, \# S_{\ell}\left(b_{\ell} ; \delta\right)<n_{\ell} / 2\right) \\
& \leqslant \phi_{0}(c, r, j) \cdot \sum_{\ell \in \Lambda_{L}} 2^{-\kappa\left(b_{\ell}, \ell ; \delta\right)},
\end{aligned}
$$

where $\phi_{0}(c, r, j)$ is a number depending only on $c, r$ and $j$. Next, as $\ell \leqslant \kappa\left(b_{\ell}, \ell ; \delta\right)$ we deduce that

$$
\forall \ell \geqslant L_{0}, \quad \boldsymbol{P}\left(C_{0}=\infty\right) \leqslant \phi_{0}(c, r, j) 2^{-L+1} .
$$

Fix a positive integer $k$ and a positive real number $\varepsilon$. For each $\ell \in \Lambda$, we set

$$
\Theta_{\ell}:=\left\{g \in \mathscr{C}^{(r)} / H_{\ell} \text { : the only representative of } g \text { in } C_{\ell} \text { is constant on } E_{\ell}^{(r)}\right\} .
$$

Of course, $\# \Theta_{\ell} / n_{\ell}=c / n_{\ell}=c^{-\left(\begin{array}{c}r+j \\ r\end{array}\right)+1}$. Before going further, we note the existence of a constant $\psi_{1}(c, r, j)$ depending only on $c, r$ and $j$ such that $\kappa\left(n_{\ell}, b_{\ell} ; \delta\right) \leqslant 8 \ell+\psi_{1}(c, r, j)$. Lemma 2.2 ensures that the hypotheses of Theorem 1.1 are satisfied. Thus, abbreviating $\kappa_{\ell}\left(b_{\ell}, \ell ; \delta\right)$ as $\kappa_{\ell}$, we obtain

$$
\begin{aligned}
\boldsymbol{P}\left(\rho_{\ell}\left(X_{k}\right) \notin \Theta_{\ell}, \forall \ell \in \Lambda_{L}\right)-\boldsymbol{P}\left(C_{0}=\infty\right) \leqslant & \left(1+c^{\left(\begin{array}{c}
r+j \\
r
\end{array}\right)}\left|\Lambda_{L}\right|\left(1-\nu \kappa_{2 L}^{-2}\right)^{k}\right)\left(\sum_{\ell=L}^{2 L} \frac{\left|\Theta_{\ell}\right|}{n_{\ell}}\right)^{-1} \\
& +\sum_{\ell=L}^{2 L} e^{-b_{\ell}} \\
\leqslant & \left(1+(L+1) \cdot c^{\left(\begin{array}{c}
r+j \\
r
\end{array}\right)}\left(1-\nu \kappa_{2 L}^{-2}\right)^{k}\right) \frac{c^{\left(\begin{array}{c}
r+j \\
r
\end{array}\right)}-1}{L} \\
& +e^{1-L}-e^{-2 L} \\
\leqslant & \frac{c^{\left(\begin{array}{c}
r+j \\
r
\end{array}\right)}}{L}+2 c^{2\left({ }_{r}^{r+j}\right)}\left(1-\nu\left(16 L+\psi_{1}(c, r, j)\right)^{-2}\right)^{k},
\end{aligned}
$$

where we use the inequality $e^{1-x}-e^{-2 x} \leqslant 1 / x$ if $x>0$. For any fixed $\varepsilon>0$, set $L:=\left\lceil k^{1 / 2-\varepsilon}\right\rceil$.

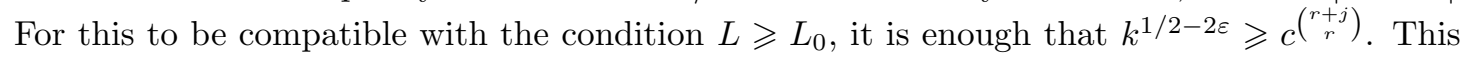
inequality can be made to hold by modifying the implied constant in the estimate proven, thereby 
making it depend also on $\varepsilon$. We compare the order of magnitude of the two summands of the above right side with the upper bound obtained for $\boldsymbol{P}\left(C_{0}=\infty\right)$. First, one has

$$
\boldsymbol{P}\left(C_{0}=\infty\right) \ll_{L_{0}} k^{-1 / 2+\varepsilon} .
$$

Also, since $L \geqslant 1$ we know that $16 L+\psi_{1}(c, r, j) \leqslant\left(16+\psi_{1}(c, r, j)\right) L$, so

$$
\begin{aligned}
\left(1-\left(16 L+\psi_{1}(c, r, j)\right)^{-2} \nu\right)^{k} & =\exp \left(-\frac{\nu}{\left(16+\psi_{1}(c, r, j)\right)^{2}} k^{2 \varepsilon}+O\left(\nu k^{-1+4 \varepsilon}\right)\right) \\
& \ll \exp \left(-\frac{\nu}{\left(16+\psi_{1}(c, r, j)\right)^{2}} k^{2 \varepsilon}\right),
\end{aligned}
$$

with an absolute implied constant. We thus obtainthe upper bound

$$
\varphi\left(\varepsilon, r, j, c, C_{0}, S(b, \delta),\left(p_{s}\right)\right) k^{-1 / 2+\varepsilon}
$$

for the probability investigated, where $\varphi\left(\varepsilon, r, j, c, C_{0}, S(b, \delta),\left(p_{s}\right)\right)$ is a positive constant depending only on the tuple $\left(\varepsilon, r, j, c, C_{0}, S(b, \delta),\left(p_{s}\right)\right)$. This finishes the proof.

Remark 1. We point out an important limitation to our approach: we cannot dispense of the use of the auxiliary parameter $j$. More precisely, letting $j$ tend to infinity in the inequality of Theorem 2.3 yields only a trivial upper bound for the probability investigated (this comes from the unboundedness of the implied constant in Theorem 2.3 as a function of $j$ ).

2.2. Monochromatic Solutions to Equations. It also seems relevant to study solutions of an equation through the perspective of Ramsey Theory: can one destroy the solutions of an equation by partitioning the different values the variables can take? We are interested in the following question, which turns out to fit our setting.

Given a random c-colouring of a random subset $A$ of $\mathbf{Z}$, what is the probability that $A$ contains a monochromatic non-empty subset summing to 0 ?

We study this question in two steps. First we leave aside colourings and just bound the probability that a random subset of $\mathbf{Z} \backslash\{0\}$ contains no subset summing to 0 . To this end, the group $G$ considered is that of all subsets of $\mathbf{Z} \backslash\{0\}$ with the symmetric difference $\Delta$ as group law. We then show how easily one can add constraints on colourings to this setting, by just considering the product of the group $G$ with the group of all $c$-colourings of $\mathbf{Z} \backslash\{0\}$. So in our setting the coloured version essentially reduces to the first question.

Let $G$ be the group consisting of all subsets of $\mathbf{Z} \backslash\{0\}$ endowed with the symmetric difference. For each positive integer $\ell$ (i.e., we choose $\Lambda:=\mathbf{N}_{\geqslant 1}$ ), we set $I_{\ell}:=\{-\ell, \ell\}, C_{\ell}:=2^{I_{\ell}}$ and we define $H_{\ell}$ to be the subgroup of $G$ consisting of all subsets of $\mathbf{Z} \backslash\{0\}$ that are disjoint from $I_{\ell}$. Thus $C_{\ell}$ forms a set of representatives for $G_{\ell}:=G / H_{\ell}$. In particular, $n_{\ell}:=\left[G: H_{\ell}\right]=4$ and $\left(H_{\ell}, C_{\ell}\right)_{\ell \geqslant 1}$ is an admissible local sequence for $G$ (since the sets $I_{\ell}$ are pairwise disjoint).

We set

$$
\Theta_{\ell}:=\left\{X \in G_{\ell}: \forall \tilde{X} \in G, \quad \rho_{\ell}(\tilde{X})=X \Rightarrow \sum_{x \in \tilde{X}} x=0\right\},
$$

so $\Theta_{\ell}$ is a singleton, the unique element of which is represented by $I_{\ell}$. Now one can define a random walk $\left(X_{k}\right)$ as in Subsection 1.3. This random walk readily satisfies the requirements of Theorem 1.1. Further, observe that if a subset $S$ of $\mathbf{Z}$ does not contain a non-empty subset summing to 0 , then neither does the intersection of $S$ with any fixed subset. Thus the probability $P_{k}$ that $X_{k}$ does not contain a non-empty subset summing to 0 is at most

$$
\boldsymbol{P}\left(\rho_{\ell}\left(X_{k}\right) \notin \Theta_{\ell}, \forall \ell\right) .
$$

We choose $b_{\ell}=\ell$ for all $\ell \in \Lambda$ and apply the same method as in the proof of Theorem 2.3 to bound $\sum_{\ell \in \Lambda_{L}} b_{\ell}$ from above. Since $\left|\Theta_{\ell}\right| / n_{\ell}=\frac{1}{4}$ for each positive integer $\ell$, Theorem 1.1 implies 
that for every positive integer $L$ and every positive integer $k$,

$$
P_{k}-\boldsymbol{P}\left(C_{0}=\infty\right) \leqslant \frac{1}{L}+\left(1+(L+1) \max _{L \leqslant \ell \leqslant 2 L}\left|G_{\ell}\right|\left(1-\nu \kappa_{2 L}^{-2}\right)^{k}\right) \cdot \frac{4}{L}=\frac{5}{L}+8\left(1-\nu \kappa_{2 L}^{-2}\right)^{k} .
$$

We have $\kappa\left(b_{\ell}, \ell ; \delta\right) \geqslant \ell$ thus $\kappa\left(b_{\ell}, \ell ; \delta\right) \geqslant n_{\ell}$ whenever $\ell \geqslant 4$. Applying Lemma 1.6 we obtain in the same way as before

$$
\boldsymbol{P}\left(C_{0}=\infty\right) \leqslant 6 \sum_{\ell=L}^{2 L} 2^{-\ell},
$$

for any choice of $L \geqslant 4$. Observing that $\kappa_{2 L} \leqslant 32 L$ by $(6)$, and setting $L:=\left\lceil k^{1 / 2-\varepsilon}\right\rceil$ we compute as in the proof of Theorem 2.3,

$$
\left(1-\nu \kappa_{2 L}^{-2}\right)^{k} \ll_{\nu} \exp \left(-\frac{\nu k^{2 \varepsilon}}{32^{2}}\right) .
$$

Consequently we infer the following statement

Theorem 2.4. Let $\left(X_{k}\right)$ be a random walk on $G$ defined as in Subsection 1.3 using $S(b, \delta)$, with $\tilde{S}_{\ell}(b, \delta) \subseteq 2^{I_{\ell}}$. Then for all $\varepsilon>0$ there exists a positive constant $C_{\varepsilon}$ (that can be computed explicitly as a function of $\varepsilon$ and $\nu$ ) depending only on $\varepsilon, S(b, \delta), C_{0}$, and the sequence $\left(p_{s}\right)$, such that for every positive integer $k$

$$
\boldsymbol{P}\left(X_{k} \text { does not contain a non-empty subset summing to } 0\right) \leqslant C_{\varepsilon} k^{-1 / 2+\varepsilon} .
$$

Let us now see how to deal with the coloured version, that is, we want to upper bound the probability that our random $c$-coloured subset does not contain a monochromatic non-empty subset summing to 0 , where $c$ is an integer greater than 1 . It suffices to work in the product group $G:=\left(2^{\mathbf{Z} \backslash\{0\}}, \Delta\right) \times\{f: \mathbf{Z} \backslash\{0\} \rightarrow \mathbf{Z} / c \mathbf{Z}\}$. For each positive integer $\ell$ (i.e., we choose $\Lambda:=\mathbf{N}_{\geqslant 1}$ ), the subgroup $H_{\ell}$ is defined to be

$$
2^{\mathbf{Z} \backslash\left(I_{\ell} \cup\{0\}\right)} \times\{f: \mathbf{Z} \backslash\{0\} \rightarrow \mathbf{Z} / c \mathbf{Z}: f(-\ell)=f(\ell)=0\}
$$

where $I_{\ell}$ is $\{-\ell, \ell\}$ as before.

Thus $n_{\ell}:=\left[G: H_{\ell}\right]=4 \cdot 2^{c}=2^{c+2}$, which does not depend on $\ell$. A set of representatives for $G_{\ell}:=G / H_{\ell}$ is $2^{I_{\ell}} \times \mathscr{F}_{\ell}$ where

$$
\mathscr{F}_{\ell}:=\left\{f: \mathbf{Z} \backslash\{0\} \rightarrow \mathbf{Z} / c \mathbf{Z}: f \mid\left(\mathbf{Z} \backslash\left(I_{\ell} \cup\{0\}\right)\right)=0\right\} .
$$

Again since the sets $I_{\ell}$ are pairwise disjoint the sequence $\left(H_{\ell}, \mathscr{F}_{\ell}\right)$ is an admissible local sequence for $G$.

Defining $\Theta_{\ell}$ to be $\left\{I_{\ell}\right\} \times\{f: \mathbf{Z} \backslash\{0\} \rightarrow \mathbf{Z} / c \mathbf{Z}: f$ is constant $\}$, it follows that $\left|\Theta_{\ell}\right| / n_{\ell}=c 2^{-c-2}$. Since the hypotheses of Theorem 1.1 are satisfied, one obtains the following statement. (The proof goes along the same lines as that of Theorem $2.4-$ in particular we choose $b_{\ell}=\ell$ and, for any fixed $\varepsilon>0$, we set $L:=\left\lceil k^{1 / 2-\varepsilon}\right\rceil$. Details are omitted.)

Theorem 2.5. Let $\left(X_{k}\right)$ be a random walk on $G$ defined as in Subsection 1.3 using $S(b, \delta)$, with $\tilde{S}_{\ell}(b, \delta) \subseteq 2^{I_{\ell}} \times \mathscr{F}_{\ell}$. Then for every positive real number $\varepsilon$ and every positive integer $k$, one has

$\boldsymbol{P}\left(X_{k}\right.$ does not contain a monochromatic non-empty subset summing to 0$) \ll_{\varepsilon} k^{-1 / 2+\varepsilon}$,

where the implied constant could be explicitly computed as a function of $\varepsilon, c$ and $\nu$ (with notation as in Theorem 1.1) and depends only on $\varepsilon, c, S(b, \delta), C_{0}$ and the sequence $\left(p_{s}\right)$.

At this point, it seems relevant to also discuss Ramsey theory for graphs. 
2.3. Looking for Monochromatic Triangles. We let $\mathscr{G}$ be the (countable) infinite complete graph, that is, the graph with vertex set $\mathbf{N}$ in which every two distinct positive integers are neighbours. We fix an integer $c \geqslant 3$ and we define $\mathscr{C}$ to be the collection of all functions from the edges of $\mathscr{G}$ to $\mathbf{Z} / c \mathbf{Z}$. As earlier, the set $\mathscr{C}$ is naturally endowed with a group structure inherited from that of $\mathbf{Z} / c \mathbf{Z}$.

We are interested in monochromatic substructures of a given fixed size that may arise. Specifically, to avoid unnecessary notation and abstraction, we shall focus on finding monochromatic triangles - though our strategy could be adapted effortlessly to the question of detecting monochromatic $r$-cliques or $r$-cycles for $r \geqslant 3$.

We define a family of subgroups $\left(H_{\ell}\right)_{\ell \in \Lambda}$ of $\mathscr{C}$, where $\Lambda:=\mathbf{N}$. Consider a partition in finite parts $\left(I_{\ell}\right)_{\ell \in \Lambda}$ of $\Lambda$. We set $i(\ell):=\left|I_{\ell}\right|$ for $\ell \in \Lambda$. Let $E_{\ell}:=\left\{(a, b) \in I_{\ell}^{2}: a \neq b\right\}$, that is, $E_{\ell}$ is the set of all edges of $\mathscr{G}$ with both endvertices contained in $I_{\ell}$. We define $C_{\ell}$ to be the collection of all functions $f \in \mathscr{C}$ with support contained in $E_{\ell}$. Then $H_{\ell}$ is the collection of all functions $f \in \mathscr{C}$ such that $f \mid E_{\ell} \equiv 0$.

Let us give the necessary properties that the quotients $\mathscr{C}_{\ell}:=\mathscr{C} / H_{\ell}$ satisfy.

Lemma 2.6. For each $\ell \in \Lambda$, the following holds.

(i) $C_{\ell}$ is a set of representatives of the quotient $\mathscr{C}_{\ell}$ and $\left(H_{\ell}, \mathscr{C}_{\ell}\right)$ is an admissible local sequence for $\mathscr{C}$; and

(ii) the index of $H_{\ell}$ in $\mathscr{C}$ is $n_{\ell}:=\left[\mathscr{C}: H_{\ell}\right]=\left|C_{\ell}\right|=c^{i(\ell)(i(\ell)-1) / 2}$.

Proof. (i) No two distinct functions in $C_{\ell}$ are congruent modulo an element of $H_{\ell}$. Moreover, for any $f \in \mathscr{C}$, let $f_{C}$ be the function equal to $f$ on $E_{\ell}$ and equal to 0 everywhere else, that is, $f_{C}\left|E_{\ell}:=f\right| E_{\ell}$ and $f_{C} \mid\left(E(\mathscr{G}) \backslash E_{\ell}\right):=0$. It follows that $f_{C} \in C_{\ell}$ and $f-f_{C} \in H_{\ell}$, or equivalently $f \equiv f_{C}\left(\bmod H_{\ell}\right)$. Finally the fact that $\left(H_{\ell}, C_{\ell}\right)$ is an admissible local sequence for $\mathscr{C}$ is a consequence of the disjointness of the sets $I_{\ell}$.

(ii) By the definition, $\left|E_{\ell}\right|=i(\ell)(i(\ell)-1) / 2$. The conclusion follows.

A practical way to rephrase part of the proof of Lemma 2.6 is to say that for each fixed integer $\ell$ in $\Lambda$ and each element $f$ of $\mathscr{C}$, the unique element in $C_{\ell}$ congruent to $f$ modulo $H_{\ell}$ is the function equal to $f$ on $E_{\ell}$ and to 0 outside of $E_{\ell}$.

From now on, we assume that $i(\ell) \geqslant 3$ for $\ell \in \Lambda$. For each integer $\ell \in \Lambda$, let $\Theta_{\ell}$ be the set of classes $\bar{f} \in \mathscr{C}_{\ell}$ such that the unique representative $f$ of $\bar{f}$ in $C_{\ell}$ (the existence of which is asserted by Lemma 2.6) contains a monochromatic triangle in $E_{\ell}$. In other words $f \in \Theta_{\ell}$ if and only if $I_{\ell}$ contains three integers $i_{1}, i_{2}$ and $i_{3}$ such that $f\left(\left(i_{1}, i_{2}\right)\right)=f\left(\left(i_{1}, i_{3}\right)\right)=f\left(\left(i_{2}, i_{3}\right)\right)$. Observe that $\left|\Theta_{\ell}\right| /\left|\mathscr{C}_{\ell}\right| \geqslant c^{-2}$. Indeed any function that restricts to a constant map (with values in $\mathbf{Z} / c \mathbf{Z}$ ) on a fixed triangle contained in $E_{\ell}$ surjects to an element of $\Theta_{\ell}$ via $\rho_{\ell}$.

Assume that $\delta$ is a fixed real number in $(0,1 / 2]$. We set $b_{\ell}:=\ell$. In particular, note that

$$
\kappa\left(b_{\ell}, \ell ; \delta\right)=\left\lceil\psi(\delta) \cdot\left(\frac{i(\ell)(i(\ell)-1) \log c}{2}+\ell+\log 2\right)\right\rceil .
$$

Given $f^{(\ell)} \in S_{\ell}\left(b_{\ell}, \delta\right)$, we define $\tilde{f}^{(\ell)}$ to be its canonical representative in $\mathscr{C}$, that is, $\tilde{f}^{(\ell)} \in C_{\ell}$. In this context, the outcome of Theorem 1.1 is the following.

Proposition 2.7. Let $\left(X_{k}\right)$ be a random walk on $\mathscr{C}$ defined as in Subsection 1.3 using $S(b, \delta)$ (see (2)) with $\tilde{S}_{\ell}(b, \delta) \subseteq C_{\ell}$. Then with notation as in Theorem 1.1, one has for any fixed positive integers $L$ and $k$

$$
\begin{aligned}
\boldsymbol{P}\left(X_{k} \text { does not contain a monochromatic triangle }\right) & \leqslant \boldsymbol{P}\left(C_{0}=\infty\right)+\frac{c^{2}+1}{L} \\
& +2 c^{(1 / 2) \cdot i(2 L)(i(2 L)-1)+2}\left(1-\kappa_{2 L}^{-2} \nu\right)^{k} .
\end{aligned}
$$


Proof. Fix a positive integer $k$. Lemma 2.6 ensures that the hypotheses of Theorem 1.1 are satisfied. We obtain, applying Theorem 1.1,

$$
\begin{aligned}
\boldsymbol{P}\left(\rho_{\ell}\left(X_{k}\right) \notin \Theta_{\ell}, \forall \ell \in \Lambda_{L}\right) & -\boldsymbol{P}\left(C_{0}=\infty\right) \leqslant\left(1+\left(\sum_{\ell \in \Lambda_{L}} n_{\ell}\right)\left(1-\kappa_{2 L}^{-2} \nu\right)^{k}\right)\left(\sum_{\ell \in \Lambda_{L}} \frac{\left|\Theta_{\ell}\right|}{n_{\ell}}\right)^{-1} \\
& +\sum_{\ell=L}^{2 L} e^{-b_{\ell}} \\
& \leqslant e^{1-L}-e^{-2 L}+\left(1+(L+1) \cdot c^{i(2 L)(i(2 L)-1) / 2}\left(1-\kappa_{2 L}^{-2} \nu\right)^{k}\right) \cdot \frac{c^{2}}{L} \\
& \leqslant \frac{c^{2}+1}{L}+2 c^{(1 / 2) \cdot i(2 L)(i(2 L)-1)+2}\left(1-\kappa_{2 L}^{-2} \nu\right)^{k},
\end{aligned}
$$

where we used that $e^{1-x}-e^{-2 x} \leqslant 1 / x$ for $x \geqslant 1$.

Different choices of sets $I_{\ell}$ may correspond to different speeds of rarefaction of non-typical structures. (We note, however, that the random walk itself does depend on the choice made for the sets $I_{\ell}$.) More precisely, one can put additional constraints on the structure of the monochromatic triangles, e.g., we may impose the three vertices to be consecutive integers as in the following theorem.

Theorem 2.8. With notation as in Proposition 2.7, one has for every positive real number $\varepsilon$ and for every positive integer $k$,

$$
\begin{aligned}
& \boldsymbol{P}\left(X_{k} \text { does not contain a monochromatic triangle }\right) \\
\leqslant & \boldsymbol{P}\left(X_{k} \text { does not contain a monochromatic triangle on three consecutive vertices }\right) \\
\ll & k^{-1 / 2+\varepsilon},
\end{aligned}
$$

where the implied constant can computed explicitly (as a function of $\varepsilon, c$ and $\nu$ (see Theorem 1.1)), and depends only on $\varepsilon, c, S(b, \delta), C_{0}$, and the sequence $\left(p_{s}\right)$.

Proof. Set $I_{\ell}:=\{3 \ell-2,3 \ell-1,3 \ell\}$ for each $\ell \in \Lambda$. In particular $i(\ell)(i(\ell)-1)=6$. Let us evaluate $\boldsymbol{P}\left(C_{0}=\infty\right)$. One has $\# S_{\ell}\left(b_{\ell} ; \delta\right) \leqslant n_{\ell}=c^{3}$ and $\kappa\left(b_{\ell}, \ell ; \delta\right)=\lceil\psi(\delta)(3 \log c+\ell+\log 2)\rceil \geqslant \ell$, by (6). In particular $n_{\ell} \leqslant \kappa\left(b_{\ell}, \ell ; \delta\right)$ for all $\ell \geqslant c^{3}$. Moreover if we assume that for all $\ell \in \Lambda_{L}$ the set $S_{\ell}\left(b_{\ell} ; \delta\right)$ contains at least $n_{\ell} / 2=c^{3} / 2$ distinct elements then $C_{0} \leqslant 2$. Therefore

$$
\begin{aligned}
\boldsymbol{P}\left(C_{0}=\infty\right) & \leqslant \boldsymbol{P}\left(\exists \ell \in \Lambda_{L}, \# S_{\ell}\left(b_{\ell} ; \delta\right)<n_{\ell} / 2\right) \\
& \leqslant\left(\begin{array}{c}
c^{3} \\
\left\lceil\frac{c^{3}}{2}\right\rceil
\end{array}\right) \sum_{\ell \in \Lambda_{L}} 2^{-\kappa\left(b_{\ell}, \ell ; \delta\right)},
\end{aligned}
$$

for all $L \geqslant c^{3}$, by virtue of Lemma 1.5.

For $\varepsilon>0$ fixed, set $L:=\left\lceil k^{1 / 2-\varepsilon\rceil}\right.$. For this to be compatible with the condition $L \geqslant c^{3}$ we need to have $k^{1-2 \varepsilon} \geqslant c^{6}$. This inequality can be made to hold by modifying the implied constant in the estimate to be proven. As in the proof of Theorem 2.3 we have

$$
\boldsymbol{P}\left(C_{0}=\infty\right) \ll_{c} k^{-1 / 2+\varepsilon} .
$$

Moreover Proposition 2.7 implies that

$\boldsymbol{P}\left(X_{k}\right.$ does not contain a monochromatic triangle on three consecutive vertices $)$

$$
\leqslant \boldsymbol{P}\left(C_{0}=\infty\right)+\frac{c^{2}+1}{k^{1 / 2-\varepsilon}}+2 c^{5}\left(1-\nu \kappa_{2 L}^{-2}\right)^{k} .
$$


To find an upper bound for the third summand we first use the assumption $L \geqslant c^{3}$ to deduce $\kappa_{2 L} \leqslant 46 L$ and then we compute

$$
\left(1-\nu \kappa_{2 L}^{-2}\right)^{k}=\exp \left(k\left(-\frac{\nu}{46^{2} k^{1-2 \varepsilon}}\right)+O\left(\nu k^{-2+4 \varepsilon}\right)\right) \ll_{c, \nu} \exp \left(-\frac{\nu}{46^{2}} k^{2 \varepsilon}\right),
$$

which finishes the proof.

We note that the contributions from the non-standard case (that is, $X\left(G / H_{\ell}, S_{\ell}\left(b_{\ell} ; \delta\right)\right)$ is not an expander) is the probability with highest order of magnitude (among the three summands in the upper bound of Theorem 1.1) given our choice of parameters in the proof of the theorem. It is natural to compare Theorem 2.8 with what is known from Ramsey theory; this discussion is deferred to the next section.

\section{Remarks and Further Applications}

As mentioned earlier, the main purpose of our work is to obtain a general sieve statement in a purely combinatorial setting. Regarding the illustrative applications, the general line of thought is to give, for the intricate notion of randomness defined, explicit upper bounds for probabilities that we expect to be small.

Let us underline some peculiarities of the application developed in Subsections 2.2 and 2.3. For monochromatic substructures, it follows from Ramsey's theorem [14] that for every fixed positive integer $c$, there exists an integer $N$ such that if $n \geqslant N$, then every $c$-colouring of the edges of the complete graph $K_{n}$ on $n$ vertices contains a monochromatic triangle. Alon and Rödl [2] established that the smallest such integer $N$ is $\Theta\left(3^{c}\right)$ as $n$ tends to infinity (that is, there exist two constants $\rho$ and $\rho^{\prime}$ such that for sufficiently large $n$, this value belongs to $\left[\rho \cdot 3^{c}, \rho^{\prime} \cdot 3^{c}\right]$ ). In our setting, although the infinite complete graph is involved, only finite subgraphs of it are checked for the existence of monochromatic triangles. These subgraphs are not necessarily large enough for Ramsey's theorem to apply. In addition, we only consider monochromatic triangles with vertices contained in some prescribed set $I_{\ell}$.

Another feature of the applications presented is uniformity of the decay rate with respect to the number $c$ of colors involved. Actually, we even claim control of the dependency of the implied constant as a function of $c$, since this implied constant could be explicitly computed. No such uniformity holds in the context of Ramsey theory. Indeed, as already mentioned, Alon and Rödl's theorem [2] asserts that the number of required vertices for Ramsey's theorem to hold grows exponentially fast with $c$.

Next let us comment on the common decay rate, roughly $1 / \sqrt{k}$, in our various applications. When applying Theorem 1.1, we always have to find an upper bound of the rough form

$$
\sum_{L \leqslant \ell \leqslant 2 L} 2^{-\ell}+c_{1} \sum_{L \leqslant \ell \leqslant 2 L} 2^{-c_{2} \ell}+\left(1+c_{3} L\left(1-\frac{c_{4} \nu}{L^{2}}\right)^{k}\right) \frac{c_{5}}{L},
$$

where each parameter $c_{i}$ is an absolute constant.

The fast decay of the first two summands is not an issue as soon as $L$ is chosen to be roughly equal to some power of $k$. However in the third summand one has to have simultaneously $L \rightarrow \infty$ and $\left(1-c_{4} \nu L^{-2}\right)^{k} \rightarrow 0$, as $k \rightarrow \infty$. These constraints justify the choice $L=\left\lceil k^{1 / 2-\varepsilon}\right\rceil$ in all our applications. There is certainly room for improvement here (e.g. by choosing a different value for $b_{\ell}$, rather than setting $b_{\ell}$ to be $\ell$, or by modifying the sieve itself so that $n_{\ell}$ is not necessarily bounded as a function of $\ell$ ), but we feel that ensuring the decay of the third summand will remain a rather serious constraint in general.

We highlight a strategy similar to that used in Subsection 2.2 that allows one to check for monochromatic arithmetic progressions for which the length, the common difference and the "shape", are prescribed. Fix positive integers $s$ (the desired length of the arithmetic progression), $q$ 
(the desired common difference), and $c \geqslant 3$ (the number of colours). Similarly as before, let $\mathscr{C}$ be the group of all $c$-colourings of $\mathbf{N}$. We consider the subsets $I_{\ell}:=\{\ell s q, \ell s q+q, \ldots, \ell s q+(s-1) q\}$ for $\ell \in \Lambda:=\mathbf{N}$. (It is this choice of particular subsets of $\mathbf{N}$ of length at least $s$ that provides a control on the "shape" of the arithmetic progressions to be found.) In this setting our method yields the following result.

Theorem 3.1. Let $\left(X_{k}\right)$ be a random walk on $\mathscr{C}$ defined as in Subsection 1.3 using $S(b, \delta)$ via the admissible local sequence $\left(H_{\ell}, C_{\ell}\right)$. For every positive real number $\varepsilon$ and every positive integer $k$,

$$
\begin{gathered}
\boldsymbol{P}\left(X_{k}\right. \text { contains no monochromatic arithmetic progression } \\
\text { with common difference } q \text { and length } s) \ll k^{-1 / 2+\varepsilon},
\end{gathered}
$$

where the implied constant could be computed explicitly as a function of $(c, s, q, \nu)$ (see Theorem 1.1 for the definition of $\nu$ ) and depends only on $(c, s, q)$, and on $C_{0}, S(b, \delta)$, and the sequence $\left(p_{s}\right)$.

Let us sketch briefly the proof. For each $\ell \in \mathbf{N}$, let $H_{\ell}$ be the set of all functions $f: \mathbf{N} \rightarrow[c]$ such that $f \mid I_{\ell} \equiv 0$. The index in $\mathscr{C}$ of each of these subgroups is $c^{s}$. Moreover, there is a collection of natural representatives $C_{\ell}$ for the classes modulo $H_{\ell}$, namely the functions with support contained in $I_{\ell}$. Thus $n_{\ell}=c^{\# I_{\ell}}$ is independent of $\ell$, and since the intervals $C_{\ell}$ are pairwise disjoint, the sequence $\left(H_{\ell}, C_{\ell}\right)$ is an admissible local sequence for $\mathscr{C}$. Let $\Theta_{\ell}$ be the set of classes modulo $H_{\ell}$ whose unique representative in $C_{\ell}$ contains a monochromatic arithmetic progression of length $s$ that is contained in $I_{\ell}$. Then one has $\left|\Theta_{\ell}\right| / n_{\ell} \geqslant c^{-s}$.

Again we may apply Theorem 1.1 with $b_{\ell}=\ell$ for all $\ell \in \Lambda$. Similarly as before, $\boldsymbol{P}\left(C_{0}=\infty\right)$ can be bounded from above: if $L \geqslant c^{(s-1) q+1}$, then $\kappa\left(b_{\ell}, \ell, \delta\right) \geqslant n_{\ell}$ whenever $\ell \geqslant L$, hence

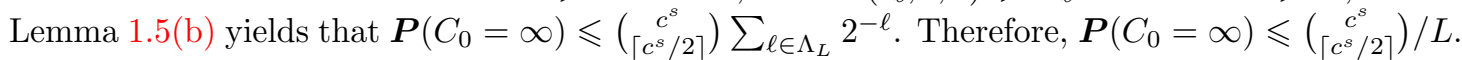

By Theorem 1.1, the probability that in $X_{k}$ no monochromatic arithmetic progression with common difference $q$ and length $s$ is contained in $I_{\ell}$, for all $\ell$ in $\Lambda_{L}$ is at most

$$
\frac{\left(\begin{array}{c}
c^{s} \\
\left\lceil c^{s} / 2\right\rceil
\end{array}\right)}{L}+\frac{1}{L}+\left(1+(L+1) c^{(s-1) q+1}\left(1-\nu \kappa_{2 L}^{-2}\right)^{k}\right)\left(c^{s} L\right)^{-1} .
$$

Since this last probability is, for every $L$, an upper bound on the probability that there is no monochromatic arithmetic progression in $X_{k}$ with common difference $q$ and length $s$, Theorem 3.1 follows by setting for any fixed $\varepsilon>0$ and $L:=\left\lceil k^{-1 / 2+\varepsilon}\right\rceil$.

We conclude by pointing out the following: van der Waerden's theorem [16] ensures that, for each fixed positive integer $s$ and each integer $c \geqslant 3$, there exists an integer $N$ such that if $n \geqslant N$ then any $c$-colouring of $[n]$ yields a monochromatic arithmetic progression of length $s$. In the above setting, we impose two additional conditions: the common difference of the arithmetic progression and a constraint on its form (it must be contained in one of the sets $I_{\ell}$ ). Van der Waerden's theorem does not guarantee the existence of such an arithmetic progression and the aforementioned inequality is essentially an explicit lower bound on the speed of rarefaction of the colourings that do not yield a monochromatic arithmetic progression with the required properties. Furthermore, and as mentioned in the remarks about Subsections 2.2 and 2.3, the uniformity of the decay rate with respect to the number of colours $c$ is a quite interesting by-product of our approach.

\section{REFERENCES}

[1] N. Alon, A. Lubotzky, and A. Wigderson, Semi-direct product in groups and zig-zag product in graphs: connections and applications (extended abstract), 42nd IEEE Symposium on Foundations of Computer Science (Las Vegas, NV, 2001), IEEE Computer Soc., Los Alamitos, CA, 2001, pp. 630-637.

[2] N. Alon and V. Rödl, Sharp bounds for some multicolor Ramsey numbers, Combinatorica 25 (2005), no. 2, $125-141$. 
[3] N. Alon and Y. Roichman, Random Cayley graphs and expanders, Random Structures Algorithms 5 (1994), no. 2, 271-284.

[4] J. Bourgain, A. Gamburd, and P. Sarnak, Affine linear sieve, expanders, and sum-product, Invent. Math. 179 (2010), no. 3, 559-644.

[5] D. Christofides and K. Markström, Expansion properties of random Cayley graphs and vertex transitive graphs via matrix martingales, Random Structures Algorithms 32 (2008), no. 1, 88-100.

[6] G. Davidoff, P. Sarnak, and A. Valette, Elementary number theory, group theory, and Ramanujan graphs, London Mathematical Society Student Texts, vol. 55, Cambridge University Press, Cambridge, 2003.

[7] F. Jouve, E. Kowalski, and D. Zywina, Splitting fields of characteristic polynomials of random elements in arithmetic groups, Israel J. Math. 193 (2013), no. 1, 263-307.

[8] E. Kowalski, The large sieve and its applications, Cambridge Tracts in Mathematics, vol. 175, Cambridge University Press, Cambridge, 2008. Arithmetic geometry, random walks and discrete groups.

[9] Z. Landau and A. Russell, Random Cayley graphs are expanders: a simple proof of the Alon-Roichman theorem, Electron. J. Combin. 11 (2004), no. 1, Research Paper 62, 6.

[10] P.-S. Loh and L. J. Schulman, Improved expansion of random Cayley graphs, Discrete Math. Theor. Comput. Sci. 6 (2004), no. 2, 523-528 (electronic).

[11] A. Lubotzky and C. Meiri, Sieve methods in group theory I: Powers in linear groups, J. Amer. Math. Soc. 25 (2012), no. 4, 1119-1148.

[12] A. Lubotzky and A. Zuk, On Property $(\tau)$, preprint, available at http://www.ma.huji.ac.il/ alexlub/ B00KS/Onproperty/Onproperty.pdf.

[13] M. Molloy and B. Reed, Graph colouring and the probabilistic method, Algorithms and Combinatorics, vol. 23, Springer-Verlag, Berlin, 2002.

[14] F. P. Ramsey, On a Problem of Formal Logic, Proc. London Math. Soc. S2-30, no. 1, 264.

[15] A. S. Golsefidy and P. P. Varjú, Expansion in perfect groups, Geom. Funct. Anal. 22 (2012), no. 6, 1832-1891.

[16] B. L. van der Waerden, Beweis einer Baudetschen Vermutung, Nieuw Arch. Wisk. 15 (1927), $212-216$.

IMB, Université de BordeAux, TAlence, France

E-mail address: florent.jouve@math.u-bordeaux.fr

C.N.R.S., LORIA, VAnderuvre-LÈS-NANCY, FranCE.

E-mail address: sereni@kam.mff.cuni.cz 